\title{
Mesenteric adipose and intestinal tissue expression and serum level of adropin in Crohn's disease patients
}

\author{
Jintong Chen ${ }^{1}$, Hongchai Xie ${ }^{1}$, Huaning Chen ${ }^{1}$, Weiwei Zheng ${ }^{1}$, Qicai Liu ${ }^{1}$, Hua Fan ${ }^{1}$, \\ Yijuan Liu ${ }^{1}$, and chengdang wang ${ }^{1}$
}

${ }^{1}$ First Affiliated Hospital of Fujian Medical University

June 2, 2020

\begin{abstract}
Background/aim: Adropin is a novel regulatory peptide that plays a vital role in lipid metabolism, insulin resistance, and adiposity. In addition, recent study has revealed that adropin appears to have anti-inflammatory properties. The main purpose of this study is to evaluate the potential association of adropin with Crohn's disease (CD) . Materials and methods: We analyzed the serum levels of adropin, inflammatory parameters, and metabolic parameters in $60 \mathrm{CD}$ patients and 36 healthy controls. Serum adropin levels were determined using a commercially available ELISA kit. Mesenteric adipose and intestinal tissues were obtained from 8 of included CD patients and, for controls, 6 colonic cancer patients who underwent colon resection. Tissue adropin expression were measured by immunofluorescence confocal microscopy. Results: Serum adropin levels were slightly lower in the active CD patients compared to the inactive CD patients $(3629.81 \pm 931.49$ versus $4322.47 \pm 1194.69 \mathrm{pg} / \mathrm{mL}, \mathrm{P}=$ 0.027). Serum adropin levels negatively correlated with ESR $(\mathrm{r}=-0.302, \mathrm{P}=0.019)$ and $\mathrm{CD}$ activity index $(\mathrm{r}=-0.332$, $\mathrm{P}$ $=0.010)$. However, mesenteric adipose and intestinal tissue adropin expression in CD patients were higher than controls, which suggests the intestine and adipose tissues may not be the major source of circulating adropin. Conclusion: Serum adropin levels decrease in the active CD patients, while adropin expression in mesenteric adipose and intestinal tissues increase in CD patients, suggesting that different source of adropin may plays a different role in CD pathogenesis.
\end{abstract}

\section{Hosted file}

Mesenteric adipose and intestinal tissue expression and serum level of adropin in Crohn's disease patie available at https://authorea.com/users/329031/articles/456160-mesenteric-adipose-andintestinal-tissue-expression-and-serum-level-of-adropin-in-crohn-s-disease-patients 